\title{
Observation and Analysis of Transequatorial Propagation
}

\author{
J. A. Thomas ${ }^{1}$ and B. A. McInnes \\ Contribution From Physics Department, University of Queensland, Brisbane
}

(Received April 21, 1964; revised July 1, 1964)

\begin{abstract}
The results of rotating backscatter sounder observations at $16 \mathrm{Mc} / \mathrm{s}$ from Brisbane are analyzed and discussed insofar as they relate to anomalous transequatorial radio-wave propagation. The association of transequatorial ground backscatter echoes with large ionospheric $F$-region tilts, which occur in equatorial regions in the evening hours, is shown. Ray-tracing calculations, for radio waves traversing these tilts, show the possibility of supermode propagation. Associated focusing effects can help explain the anomalous signal strength of the recorded echoes. The need for careful interpretation of backscatter echoes, in the presence of a number of propagation modes, is stressed. Comparisons of the results of the ray tracings and the experimental records are made for the 6th October, 1960, and for the average conditions of September 1960. In both cases reasonable agreement is obtained.
\end{abstract}

\section{Introduction}

Over the past few years, interest has been stimulated in the investigation of transequatorial propagation on account of the unexpectedly high signal strength of long-distance transmission (up to 10,000 $\mathrm{km}$ ) in this region, as reported by Villard, Stein, and Yeh [1957] and Dueno [1960]. Suggested explanations have been put forward [Stein 1958, Dueno 1960, Kift 1960, Muldrew and Maliphant 1962] for the phenomenon. Since September 1960, regular rotating backscatter sounding observations have been carried out at Brisbane on a frequency of 16 $\mathrm{Mc} / \mathrm{s}$ using a narrow beam antenna [Thomas and McNicol, 1960]. In this paper some of the observations made in the first year of recording will be discussed. By ray-tracing computations, based on ionospheric configurations derived indirectly, it has proved possible to interpret results as tilt-mode propagation in some typical cases.

\section{Observations of Transequatorial Radio Propagation From Brisbane}

\subsection{Backscatter Soundings}

In figure 1 is shown a series of tracings of $16 \mathrm{Mc} / \mathrm{s}$ backscatter records at Brisbane. The tracings were made at two-hourly intervals, and the days illustrated are chosen from the two-monthly periods used in later analysis, viz, September-October, 1960, November-December, 1960, January-February, 1961, March-April, 1961, May-June, 1961, and July-August, 1961. The broken curve shown in each $0100 \mathrm{hr}$ plot represents the ground-range

Present address: School of Physics (R.A.A.F. Academy), University of Melbourne. azimuth plot of the magnetic equator and serves as a guide to judging which propagation path actually crossed the equator. All times are local standard times for Brisbane.

A striking feature, shown on several of the late afternoon plots of figure 1 , is the patch of echoes extending to great ranges in the northeast. Detailed examination of the records shows that this patch typically appears first at an azimuth of 60 to $70^{\circ}$ as a weak echo at approximately 9 to $10,000 \mathrm{~km}$ range. It gradually extends in azimuth (particularly toward the north), and almost invariably has the appearance of the right arm of a $U$ on the rangeazimuth plot. This type of patch appears to be comparatively stable, sometimes remaining in much the same position for 3 to 4 hrs, whilst the patches corresponding to normal second-hop and third-hop propagation (which show as horizontal bands), change considerably. An example of such a sequence has been published elsewhere [Thomas, 1961].

After September, 1961, hourly measurements were made of the relative strength of echoes in the various modes of propagation using the swept-gain technique; prior to that data only spasmodic measurements were made. The range of settings available during the sweep was $66 \mathrm{~dB}$ and readings were accurate only to $6 \mathrm{~dB}$. The minimum detectable signal at the receiver was about $0.2 \mu \mathrm{V}$, and a total transmission loss of about $200 \mathrm{~dB}$ gave echoes barely detectable in the records (with $5 \mathrm{~kW}$ peak pulse power).

Signal strengths of one-hop $(1 \mathrm{~F})$ echoes were normally 30 to $60 \mathrm{~dB}$ above threshold. The signal strength of the anomalous long-range transequatorial echoes (T.E.) ${ }^{2}$ was typically 18 to $30 \mathrm{~dB}$ weaker than the simultaneous $1 \mathrm{~F}$ echo in the same direction.

2 The Classification “T.E." is reserved for those transequatorial echo patches. which indicate apparent anomalous propagation, and as far as possible excludes normal $2 \mathrm{~F}, 3 \mathrm{~F}$, or $4 \mathrm{~F}$ patches even if transequatorial. 


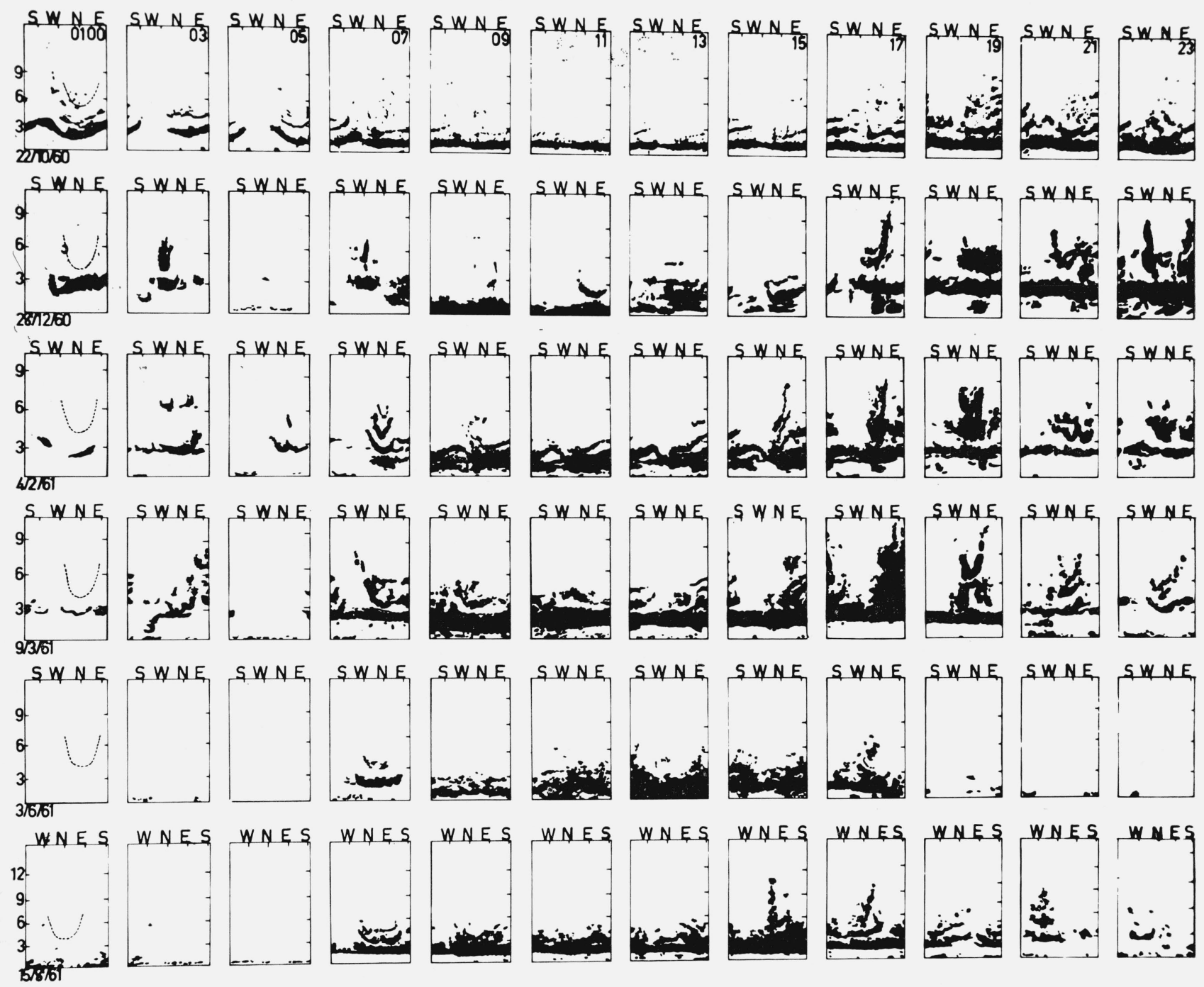

FIGURE 1. $16 \mathrm{Mc} / \mathrm{s}$ backscatter records obtained at Brisbane on representative days throughout a year.

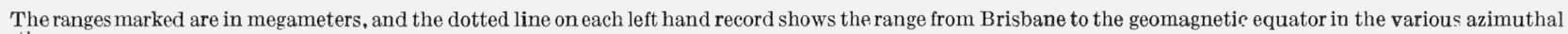
directions.

When the patch was spread greatly in range and azimuth, the intensity was only a few $\mathrm{dB}$ above threshold. On the other hand, on occasion, intensities only $6 \mathrm{~dB}$ below $1 \mathrm{~F}$ were found, even for echoes at a range nearly 8 times that of the $1 \mathrm{~F}$ echoes, suggesting a considerable degree (10 $\mathrm{dB}$ or more) of focusing of energy.

Transequatorial echoes differ in character from normal (1F, etc.) backscatter echoes in that they rarely show a well-defined leading edge, and that various portions of the echo fade independently of one another. The outline is usually "fuzzy," though the fading rate is not exceptionally high. On the other hand, phase-path (Doppler) observations show no phase coherence for T.E. echoes, whereas $1 \mathrm{~F}$ echoes may show coherence lasting for several seconds at a time in the leading edge of the returned echo.

\subsection{Analysis of Results}

For statistical purposes it has been found convenient to group together echoes recorded at $20^{\circ}$ intervals of aximuth, commencing at $70^{\circ} \mathrm{E}$ of North and traversing through North to $290^{\circ}$. All directions are magnetic bearings from Brisbane. For each direction of observation considered, the presence or absence of $E_{s}, 1 \mathrm{~F}, 2 \mathrm{~F}, 3 \mathrm{~F}$ and transequatorial (T.E.) mode echoes was noted at half-hourly intervals throughout the day, for the year September 1960, to August 1961.

Whilst it is usually a simple matter to distinguish a T.E. echo at $70^{\circ}$ and $50^{\circ}$, this echo tends to merge into normal mode echoes nearer to the north, where its range is less. Often the assignment of a particular patch to the T.E. classification is only justified by its apparent continuity and previous history at 

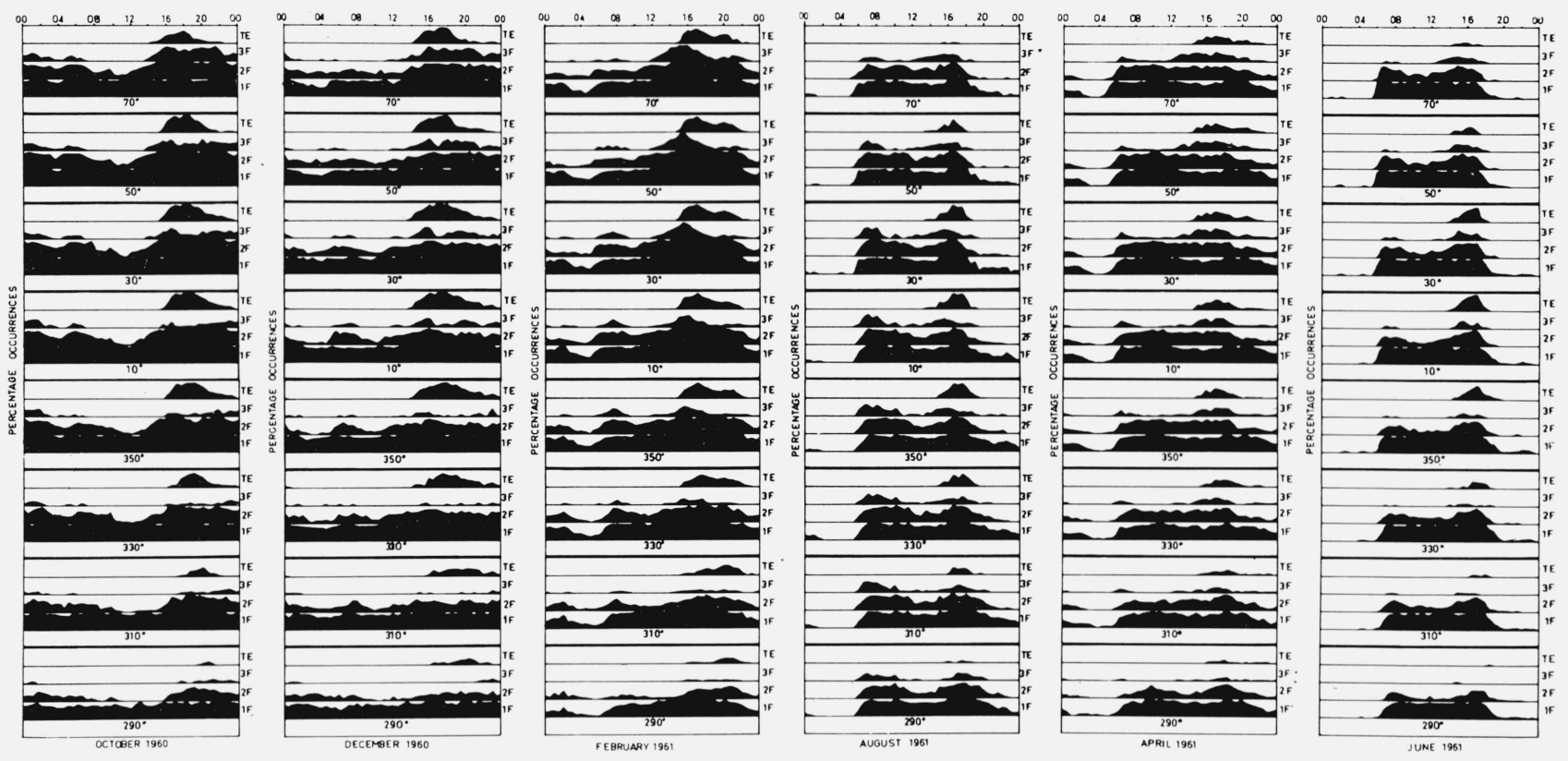

Figure 2. Variations of $16 \mathrm{Mc} / \mathrm{s}$ backscatter echoes propagated via $1 \mathrm{~F}, 2 \mathrm{~F}, 3 \mathrm{~F}$, and T.E. modes for $1960-61$.

other azimuths. The data presented may therefore contain a few faulty identifications. It will be shown later that a T.E. echo is very often complex, with components from a variety of paths.

An assessment was then made for each hour, (when the system was operational), and for each interval of azimuth, as to whether a $1 \mathrm{~F}, 2 \mathrm{~F}, 3 \mathrm{~F}$, or 'T.E. echo was present, or not. From the aggregate of results for each month, percentage occurrences were determined. These are shown in figure 2. (All times are local times, and the vertical interval for each plot represents 100 percent occurrence.)

The outstanding features shown by these plots are:

(a) T.E. echoes occur almost exclusively between the hours of 1400 and 0000 . More of these echoes occur to the east of north than to the west, with peak occurrence at 10 to $30^{\circ}$. They tend to occur relatively early in the east and progressively later for sectors further towards the west. The occurrence is spread over more of the day in summer than in winter.

(b) The presence of T.E. echoes sometimes seems to be associated with the absence of $3 \mathrm{~F}$ echoes particularly for the summer months and northeasterly bearings. On these occasions it may well be that the normal $2 \mathrm{~F}$ echo is missing rather than the $3 \mathrm{~F}$ echo. This uncertainty can only be resolved by far more accurate and painstaking scaling of the records than has yet been possible. This question of accurately identifying the various modes of propagation will be taken up again later.

(c) In all months T.E. echoes are absent, or rare, at hours when $1 \mathrm{~F}$ (and to a lesser extent $2 \mathrm{~F}$ ), echoes are rare. The situation for June is especially striking in this regard.

(d) The regular progression of occurrence times of T.E. echo patches with change in bearing seems to imply some special ionospheric feature moving from east to west in the late afternoon and evening hours (Brisbane time) and capable of giving rise to the anomalous echoes observed. Such a feature is in fact found in the height variations of the $F$-layer over equatorial regions and is discussed later.

(e) No plots for $E_{s}$ echoes are shown in figure 2 . There appears to be no correlation (either short-term or long-term) between the presence of $E_{s}$ backscatter echoes and the occurrence of T.E. echoes, and indeed, the very stability of the T.E. echoes in the northeasterly direction argues against such an erratic feature as sporadic- $E$ playing an important part in their appearance. Kift [1960] found that only equatorial $E_{s}$ was of importance in regard to such propagation paths and this type of $E_{s}$ disappears before the anomalous T.E. echoes occur [Smith, 1957].

\subsection{Range Variation of Backscatter Echoes}

If the position of the ground-scattering region for the T.E. echoes is deduced from the range and azimuth recorded, it is found to be roughly an east-west zone, a few degrees wide, approximately centered on geomagnetic latitude $20^{\circ} \mathrm{N}$. The temporal variation of the range of the T.E. echoes, in the direction of minimum range (approximately magnetic north) for the period 14th September, 1961 to 12 th October, 1961 , is shown in figure 3 . The standard variation of the means is shown as a series of vertical bars. (The minimum range of the $20^{\circ} \mathrm{N}$ line of magnetic latitude from Brisbane is $5700 \mathrm{~km}$.) There appears to be a significant change in the minimum range observed as time progresses. 


\section{Ionospheric Height and Frequency Plots}

\subsection{General Method of Analysis}

There is a great dearth of ionospheric stations in the area to the north and east of Brisbane; this means that the detailed behavior of the ionosphere in these regions is not available for comparison with backscatter soundings.

However, the worldwide pattern of behavior of $f_{0} F_{2}$ and $h^{\prime} F$ becomes comparatively simple when plotted in geomagnetic coordinates [Martyn, 1955], and equatorial sporadic- $E$ exists only over a narrow belt close to the magnetic equator [Smith, 1957]. It is therefore feasible to attempt some projection of ionospheric behavior patterns, making due allowance for difference in local time, along the lines of magnetic latitude, and so obtain a first approximation to the conditions in regions where no verticalincidence sounder exists.

Sources of $F$-region data were the $F$-series publications of the Central Radio Propagation Laboratory and Daily Data Sheets of the Ionospheric Prediction Service of Australia and the data sheets of the Radio Research Laboratories of Japan.

Data were used from the following stations:

\begin{tabular}{|c|c|}
\hline Station & $\begin{array}{c}\text { Geomagnetic } \\
\text { latitude }\end{array}$ \\
\hline $\begin{array}{l}\text { Canberra } \\
\text { Mundaring } \\
\text { Brisbane } \\
\text { Townsville_. } \\
\text { Buenos Aires } \\
\text { Rarotonga } \\
\text { Singapore } \\
\text { Huancyao } \\
\text { Talara } \\
\text { Taipei } \\
\text { Yamagawa } \\
\text { Kokubunji } \\
\text { El Cerrillo } \\
\text { Akita } \\
\text { Wakkanai }\end{array}$ & $\begin{array}{l}-44^{\circ} \\
-41^{\circ} \\
-36^{\circ} \\
-28^{\circ} \\
-23^{\circ} \\
-20.7^{\circ} \\
-10^{\circ} \\
\quad-1^{\circ} \\
+6.6^{\circ} \\
+14^{\circ} \\
+20.3^{\circ} \\
+26^{\circ} \\
+29.2^{\circ} \\
+29.5^{\circ} \\
+35^{\circ}\end{array}$ \\
\hline
\end{tabular}

Since T.E. echoes appear mainly in the late afternoon and evening, projection of $f_{0} F_{2}$ and $h^{\prime} F$ data was carried out for Brisbane times 1500, 1700, 1900, and $2100 \mathrm{hr}$. Thus, for example, data from each available station at $1500 \mathrm{hr}$ local time were placed on a map at the geomagnetic latitude appropriate to the station, and on the $150^{\circ} \mathrm{E}$ (geographic) meridian. Similarly, $1600 \mathrm{hr}$ values were entered on the $165^{\circ} \mathrm{E}$ meridian, $1700 \mathrm{hr}$ values on the $180^{\circ}$ meridian, and so on. In some cases (e.g., Akita and El Cerrillo) data from two stations falls on almost the same position on the map; on most of such occasions, reasonable agreement between the two sets of data is obtained. In this way, a set of values is obtained through which it is possible to draw "contour lines" of $f_{0} F_{2}$ and $h^{\prime} F$ for $1500 \mathrm{hr}$ Brisbane time.

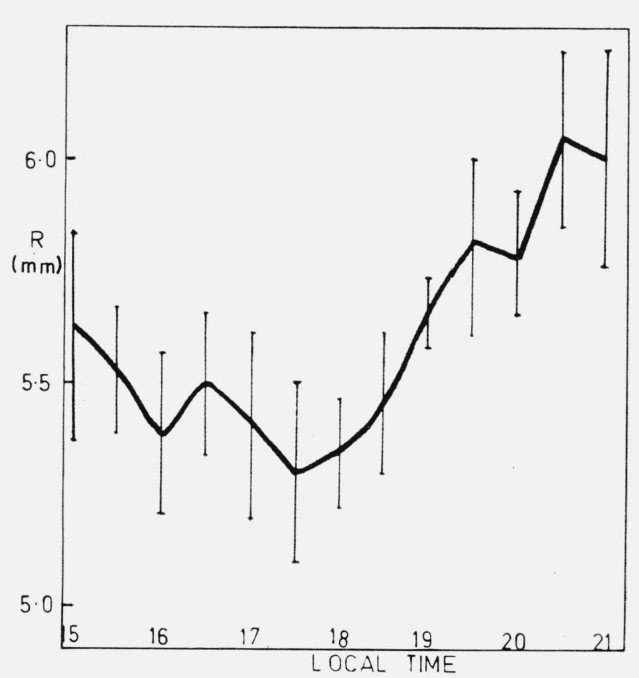

Figure 3. Temporal variation of the minimum range of anomalous $16 \mathrm{Mc} / \mathrm{s}$ transequatorial backscatter echoes.

By drawing appropriate great circle lines on the "contour" map it was then possible to estimate the variation of $f_{0} F_{2}$ and $h^{\prime} F$ with range along a series of great circles through Brisbane at $1500 \mathrm{hr}$ Brisbane time. A corresponding procedure was carried out for the other Brisbane times analyzed. ${ }^{3}$

\subsection{Virtual Height Plots}

Previous work [Villard et al., 1957; Dueno, 1960) indicated that observation of anomalous (tilt-mode) propagation is most intimately associated with the height variation of the $F$-layer across equatorial regions. For this reason, a more extensive analysis has been made of $h^{\prime} F$ than of $f_{0} F_{2}$.

In figures 4 and 5 are shown sample plots of monthly median values of $h^{\prime} F$ for each season between March, 1959 and March 1961. In each of these plots, the horizontal axis represents 10,000 $\mathrm{km}$ of range.

The most striking feature of these plots is the regular progression of the high peak of $h^{\prime} F$ values from $70^{\circ}$ at $1500 \mathrm{hr}$ to $310^{\circ}$ at $2100 \mathrm{hr}$. This is a consequence of the method of deriving these contours; the equatorial bulge in ionospheric heights moves around the world as time progresses and thus changes in distance and direction from Brisbane.

In the direction $290^{\circ}$ from Brisbane the only apparent systematic variation which exists is that of a general decrease of $h^{\prime} F$ with range from Brisbane. This is interesting in view of the generally low occurrence of transequatorial echoes in this direction as compared to the other directions analyzed.

For some hours prior to the passage of the equatorial bulge through a particular great circle, there is a general tendency for the slope in the $h^{\prime} F$ plot

${ }^{3}$ In later work, because of the availability of data from additional stations, it has been possible to restrict projection to a maximum of $45^{\circ}$ longitude on each side of the station. In addition, magnetic control has been substituted for geomagnetic control. 


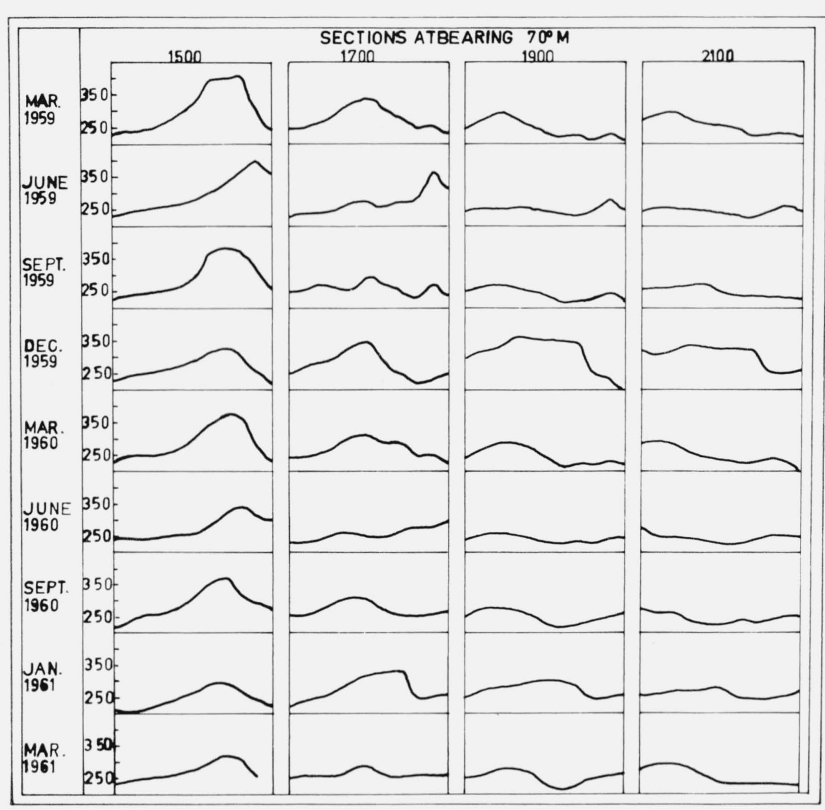

Figure 4. Sectional plots of median h'F values for a great circle path leaving Brisbane at a magnetic bearing of $70^{\circ}$.

In each plot Brisbane is at the left-hand end of the plot, the horizontal axis represents $10,000 \mathrm{~km}$ of ground range, and the vertical axis represents virtua height.

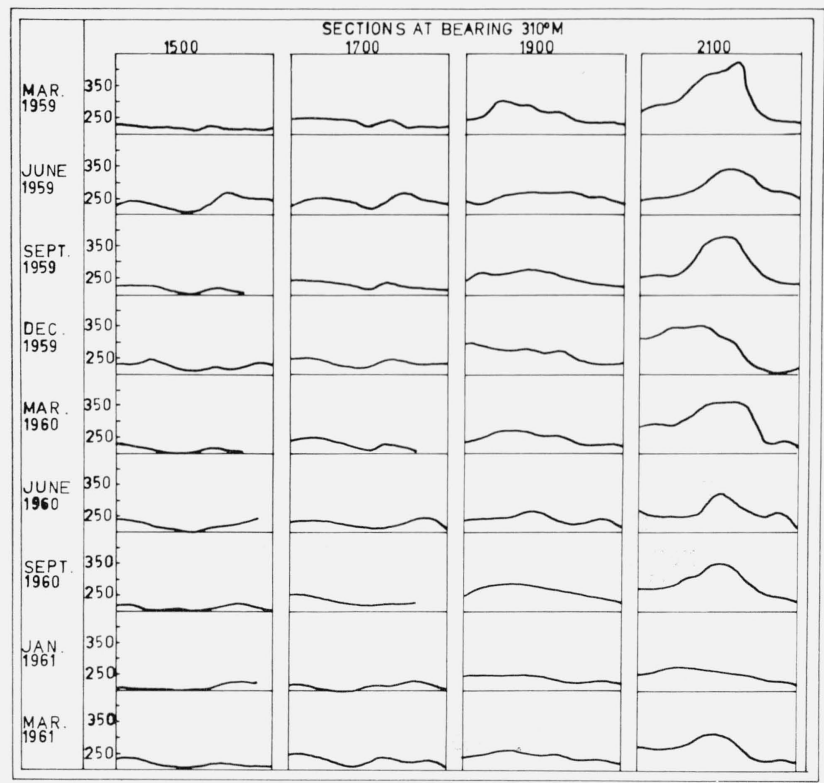

Figure 5. As for figure 4, with a magnetic bearing of $310^{\circ}$.

to be downwards, at least initially. Thus the 1500 $\mathrm{hr}$ plots and, to some extent, the $1700 \mathrm{hr}$ plots for bearings of $10^{\circ}, 350^{\circ}, 330^{\circ}, 310^{\circ}$, and $290^{\circ}$ show cases of height decreasing with range. The same tendency is shown a few hours after the bulge has passed, e.g., the $2100 \mathrm{hr}$ plots for $70^{\circ}$ (i.e., about $6 \mathrm{hr}$ after the passage of the bulge) show several cases of height decreasing with range. Negative tilts of this nature in the height of the $F$-region might be expected to reduce the possibility of tilt-mode propagation. This is in broad agreement with the fact that at about $2100 \mathrm{hr}$ the echo pattern tends to become very confused in the north and east, and that T.E. echoes tend to occur to the north and west at later times than in the east. No clear-cut relationship exists however, and this point will be discussed further later.

During northern summer months there is a pronounced positive tilt in the $F$-region height profiles, with the northern hemisphere $F$-region virtual heights some $40 \mathrm{~km}$ higher than those of the southern hemisphere. There appears to be no substantial tendency for this situation to reverse during southern summer.

\subsection{Critical Frequency Plots for the F-Region}

Samples of the azimuth plots for $F_{2}$ region critical frequencies are shown in figures 6 and 7 . At the equinoxes (and to a lesser extent during Southern summer), two maxima tend to appear in the $f_{0} F_{2}$ profiles, one on either side of the geomagnetic equator, as expected [Martyn 1959]. At the northern solstice and in equinoctial months, the positions of the peaks, while naturally dependent on direction, do not depend on the hours of day e.g., the closer peak for $20^{\circ}$ during April 1961 remains at $3000 \mathrm{~km}$ from 1700 to $2000 \mathrm{hr}$. During the southern solstice the position and magnitude of the peak of $f_{0} F_{2}$ depends upon both hour of day and azimuth. Thus at $60^{\circ}$ in December 1960 , the peak $f_{0} F_{2}$ value shifts in range from about $8000 \mathrm{~km}$ at $1600 \mathrm{hr}$ (not shown), through $4500 \mathrm{~km}$ at $1800 \mathrm{hr}$ and $3500 \mathrm{~km}$ at $2000 \mathrm{hr}$, to $2500 \mathrm{~km}$ at $2200 \mathrm{hr}$ (not shown), and the $20^{\circ}$ profile shows much the same effect. It thus appears that the time sequence of behavior of the electron density of the $F$-region along transequatorial paths cannot be the prime cause of the observed anomalous transequatorial echoes, which show for all seasons a significant time sequence of occurrence.

However, one feature of the T.E. echoes patterns can be explained by the $f_{0} F_{2}$ variations. Until about $1800 \mathrm{hr}$, at all seasons, the value of $f_{0} F_{2}$ for the first few thousand kilometers is sufficient to support the propagation of $16 \mathrm{Mc} / \mathrm{s}$ signals. After $1800 \mathrm{hr}$ in June, the critical frequency drops to a value too low to support propagation at this frequency, first along easterly directions and progessively later in northerly and westerly directions, thus accounting for the cessation of backscatter signals after 1800 for June as in figure 2.

\section{Ray Tracing}

\subsection{Method Employed}

In the previous sections it has been shown that a correlation appears to exist between the equatioral bulge in $F$-region height and the occurrence of T.E. echoes. In order to determine whether the effect of the equatorial bulge on radio waves is such as to give T.E. echoes at the ranges observed and with the 

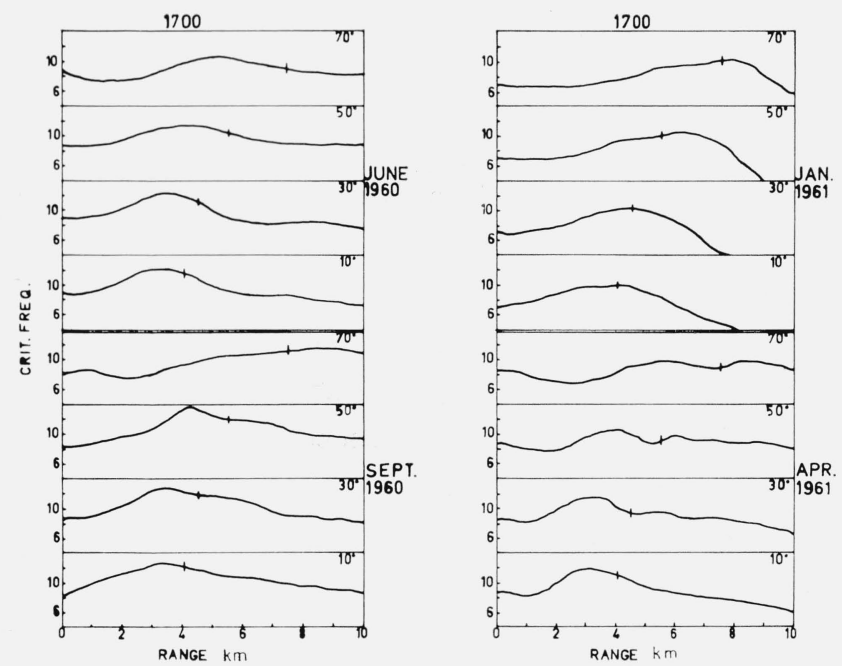

Figure 6. Sectional plots of median $\mathrm{f}_{0} \mathrm{~F}_{2}$ values for great circle paths leaving Brisbane at magnetic bearings of $\mathrm{\gamma O}^{\circ}$, $50^{\circ}, 30^{\circ}$, and $10^{\circ}$.

The horizontal axis represents $10,000 \mathrm{~km}$ of ground range, and the vertical axis represents critical frequency
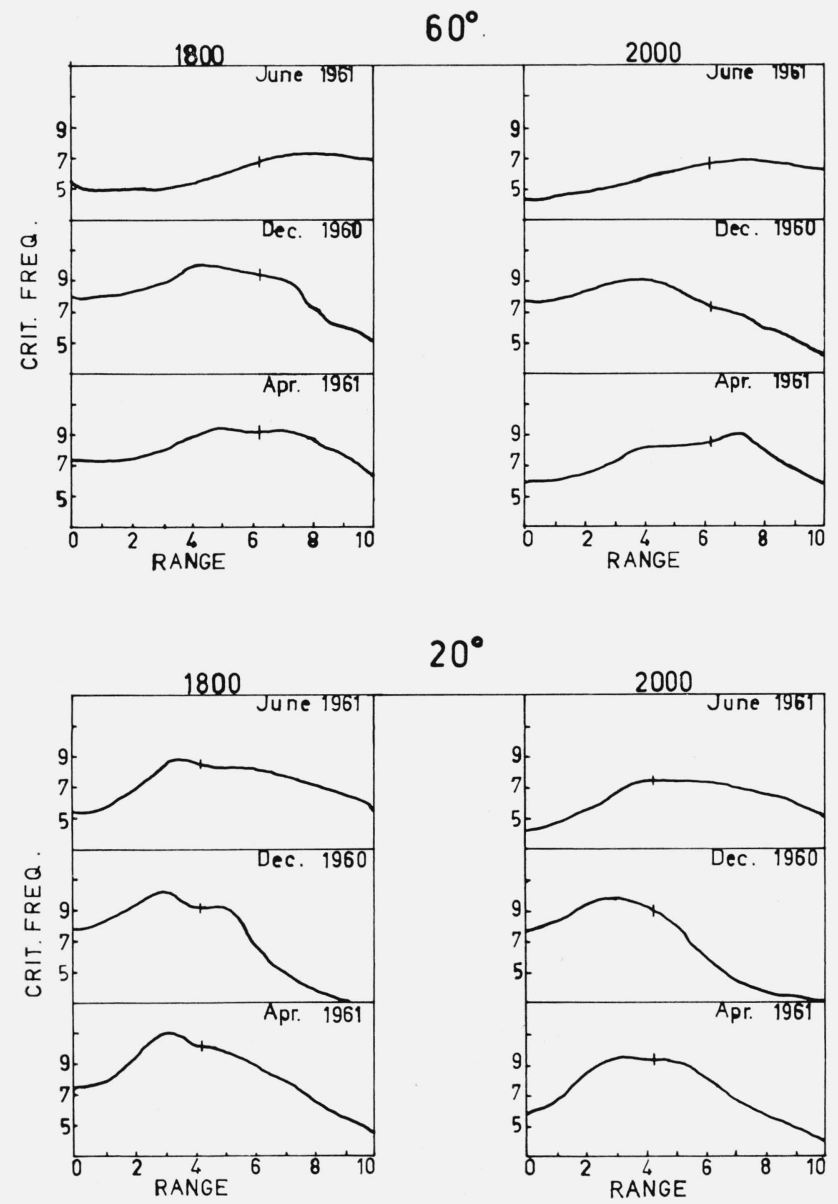

Figure 7. As for figure 6 , at magnetic bearings of $60^{\circ}$ and $20^{\circ}$. anomalous signal strength recorded, ray tracing procedures have been applied, taking a series of elevation angles up to penetration for the initial ray, and repeating for a series of different azimuths. Three procedures have been employed.

(a) A fully graphical method based on a plot of refractive index contours, with a scale of $40 \mathrm{~km}$ equals 1 in., along a vertical plane at each selected azimuth. In some cases this plot could be obtained from ionograms, but mostly it was necessary to assume a parabolic layer and employ the method of Rawer and Taieb [1960] to obtain the relevant data. The ray paths were drawn, assuming discontinuous refractive index jumps of 0.01 , and assuming the ray to be coincident with the wave normal, so that Snell's Law applied. To determine the top of the path, the procedure of Rossi [1957] was employed. Full allowance was made for the curvature of the earth. In this method an initial error of $1 / 4^{\circ}$ may result in an error of several hundred kilometers for long-range echoes.

(b) A partly computational method, in which the range paths were computed below $250 \mathrm{~km}$, (or at the base of the ionosphere, if below $250 \mathrm{~km}$, and found graphically, as before, above this height. In this case the scale used was $5 \mathrm{~km}$ equals 1 in., but the errors in angular measurement were not reduced.

(c) A fully computational method approximating to that of Kift [1960] except that allowance was made for the skewness of the ionosphere. Utilizing the great circle profiles previously derived, tilts are included by correction of the angle of incidence at the base of the ionosphere, for entry into and exit from the layers. A minor variation from the analysis of Kift is that the semithickness is assumed constant at $150 \mathrm{~km}$, on the basis of the profiles of Wright [1960]. To facilitate calculations, numerous largescale graphs were constructed relating the relevant variables. Where supermodes were indicated, i.e., where the ray path was "reflected" twice from the ionosphere, without intermediate ground reflection, a slight modification of the method was necessary, but the graphs could still be employed.

Calculations were made of group path length (corresponding to the range as determined from backscatter records), using an adaption of Shearman's [1956] method taking account of the asymmetry of the path. Full details of these methods are given elsewhere [Thomas and McInnes, 1962].

\subsection{Mode Changing}

If signals arrive at a common ground point by two different propagation paths $\mathrm{A}$ and $\mathrm{B}$, then a ray arriving by path $\mathrm{A}$ and scattered at the ground would be expected to return by both paths A and B. Since the time delays associated with the two paths are usually different, separate echoes should be received with delays corresponding to $2 \mathrm{~A}, 2 \mathrm{~B}$, and $\mathrm{A}+\mathrm{B}$. This process is important as a means of transferring power form high-angle modes to lowangle modes and thus enhancing signal power in otherwise weak backscatter echoes. 


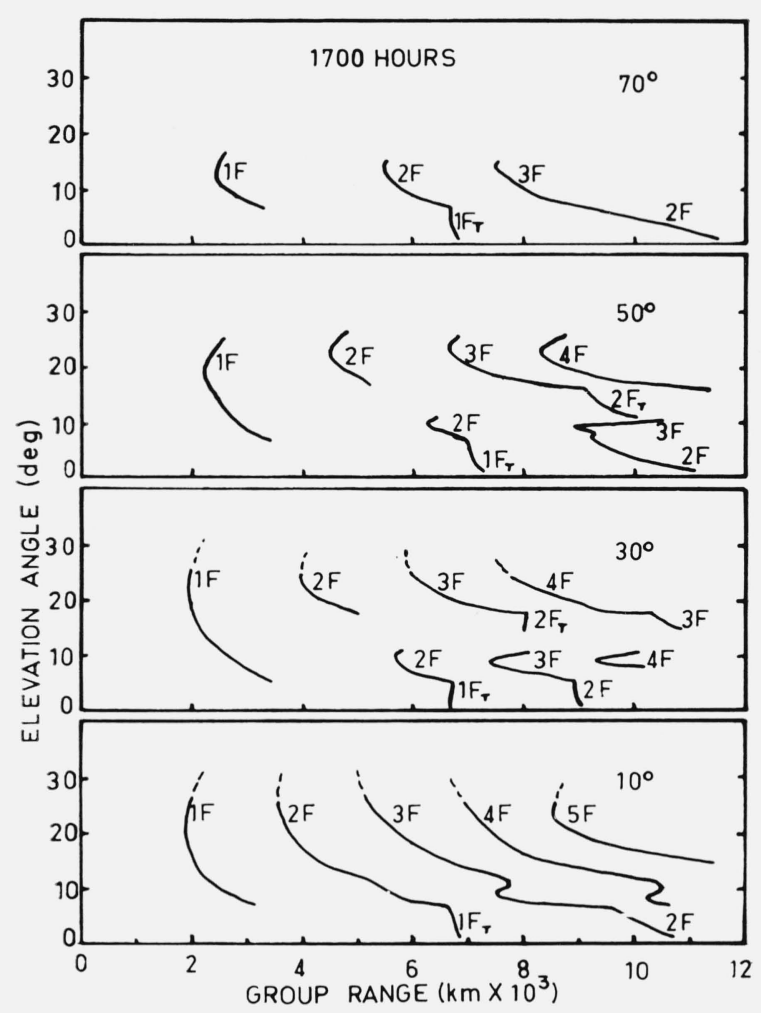

Figure 8. Mode-angle plots for $16 \mathrm{Mc} / \mathrm{s}$ waves leaving Brisbane on magnetic bearings of $70^{\circ}, 50^{\circ}, 30^{\circ}$, and $10^{\circ} \mathrm{E}$ of North derived using Median values of $\mathrm{h}^{\prime} \mathrm{F}$ and $\mathrm{f}_{0} \mathrm{~F}_{2}$ for September 1960.

\subsection{Ray-Tracing Results}

Following Kift [1960], results obtained by the methods outlined above, omitting the mixed modes of 4.2 , have been plotted in terms of elevation angle against group range (fig. 8). This presentation enables rapid assessment of the relative importance of the various modes in producing echoes at any given range. In some of the cases represented there is a considerable overlap of propagation modes in ground range and the number of "mixed-mode" signals which could be received is large. Great caution must be exercised in interpreting backscatter records is such cases.

An important feature of the mode-angle diagrams is that the group range sometimes varies very little over a considerable range of elevation angles (usually for low angles) i.e., focusing exists. In such cases, small changes in the ionization profiles are found to have little effect on the range of the echoes. It may therefore be anticipated that such focusing effects would not only enhance the intensity of the echoes, but also their stability.

\subsection{Relative Signal Strengths of Backscatter Echoes}

The relative signal strengths of backscatter echoes, found at various group ranges in the mode- angle diagrams, obtained as above, depend on:

(1) distance attenuation,

(2) focusing due to ionospheric and ground curvature,

(3) nondeviative absorption,

(4) ground reflections,

(5) antenna radiation pattern,

(6) ground backscatter coefficients, and

(7) deviative absorption.

By estimating these factors, it is possible to ascribe relative signal strength figures to the various modes and combinations of modes. Full details are given elsewhere [Thomas and McInnes, 1962].

At this point it would be preferable to have experimental results (such as obtained by sweptgain records) to compare with the results of the ray tracing and signal strength analysis. The lack of swept-gain records during the period investigated here (September 1960 to August 1961) prohibits this approach. However, it is possible to build up echo plots (range against signal strength curves) on the basis of the ray tracing, and, after estimating the threshold level of recording, to compare analytical with experimental results. The latter indicates the presence of stable, somewhat isolated, echo patches at ranges from 5,000 to $10,000 \mathrm{~km}$. Such scant signal strength information as is available, indicates that these echoes have greater signal strength than would be expected from normal $3 \mathrm{~F}$ and $4 \mathrm{~F}$ echoes at similar ranges.

Relative signal strength plots and backscatter records were compared for $50^{\circ}$ and $70^{\circ}$ magnetic azimuth at $1600 \mathrm{hr}$, 6th October 1960. Figure 9 indicates that calculations based on an inverse distance attenuation did not indicate the existence of echoes of intensity sufficient to appear above the baseline at ranges of 9,000 to $11,000 \mathrm{~km}$, where strong echoes were in fact recorded. Lowering of the baseline (which assumes increased intensity of

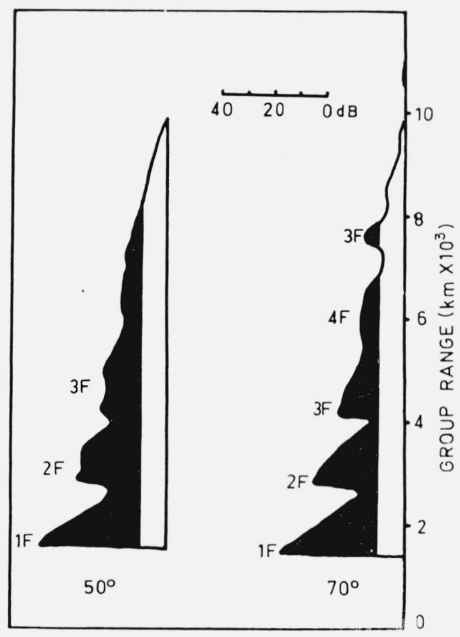

FIgure 9. Plots of the computed relative field strength of echoes at $50^{\circ}$ and $70^{\circ}$ for $1600 \mathrm{hr}$, 6th October, 1960 .

Inverse distance attenuation was used in the computations. 


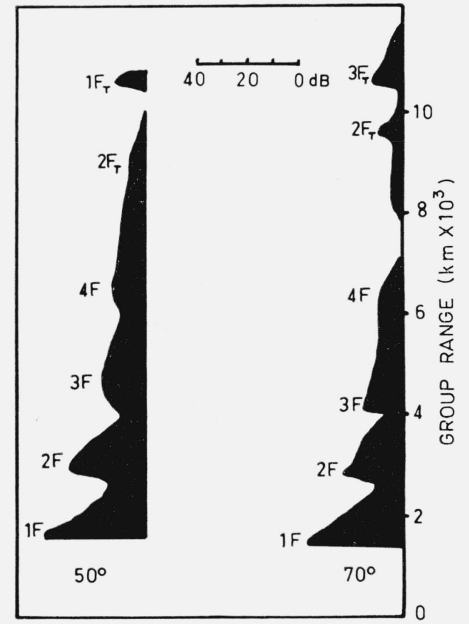

Figure 10. As for figure 9, but with horizon focusing effects included in the computation.

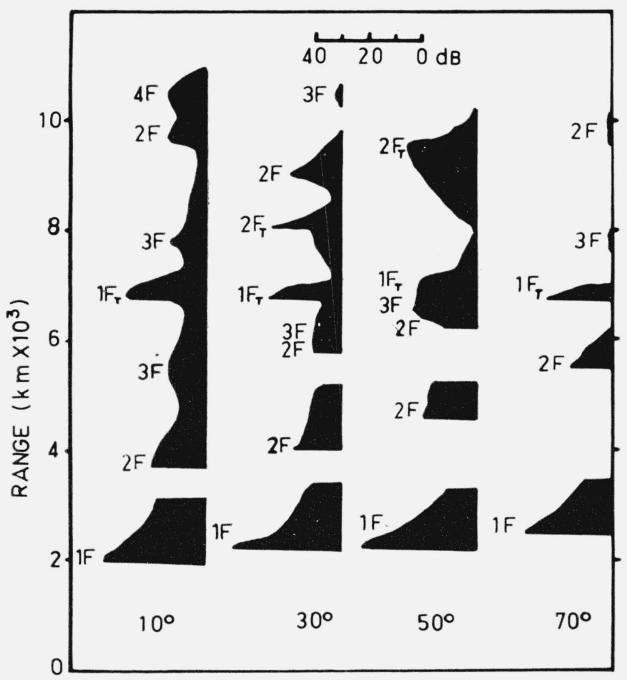

Figure 11. Plots of the computed relative field strength of echoes from the mode-angle plots of figure 8 (median values for September 1960).

the strongest echo) is not sufficient to account for this failure, because the echoes then noted are not isolated, but correspond to continuous echo reception out to a range of $11,000 \mathrm{~km}$. However, when horizon focusing effects [Rawer, 1952] are taken into account, reasonably strong isolated echoes are obtained on the signal strength plots (fig. 10).

Rawer [1952] and Gerson [1962] have already noted the breakdown of the inverse distance attenuation in failing to explain the existence of antipodal echoes. Piggott [1953] also noted that, because of horizon focusing, the total spatial attenuation may in certain cases decrease with increasing distance beyond a certain range.

A further comparison was made using echo plots on the median ionospheric data of September 1960.

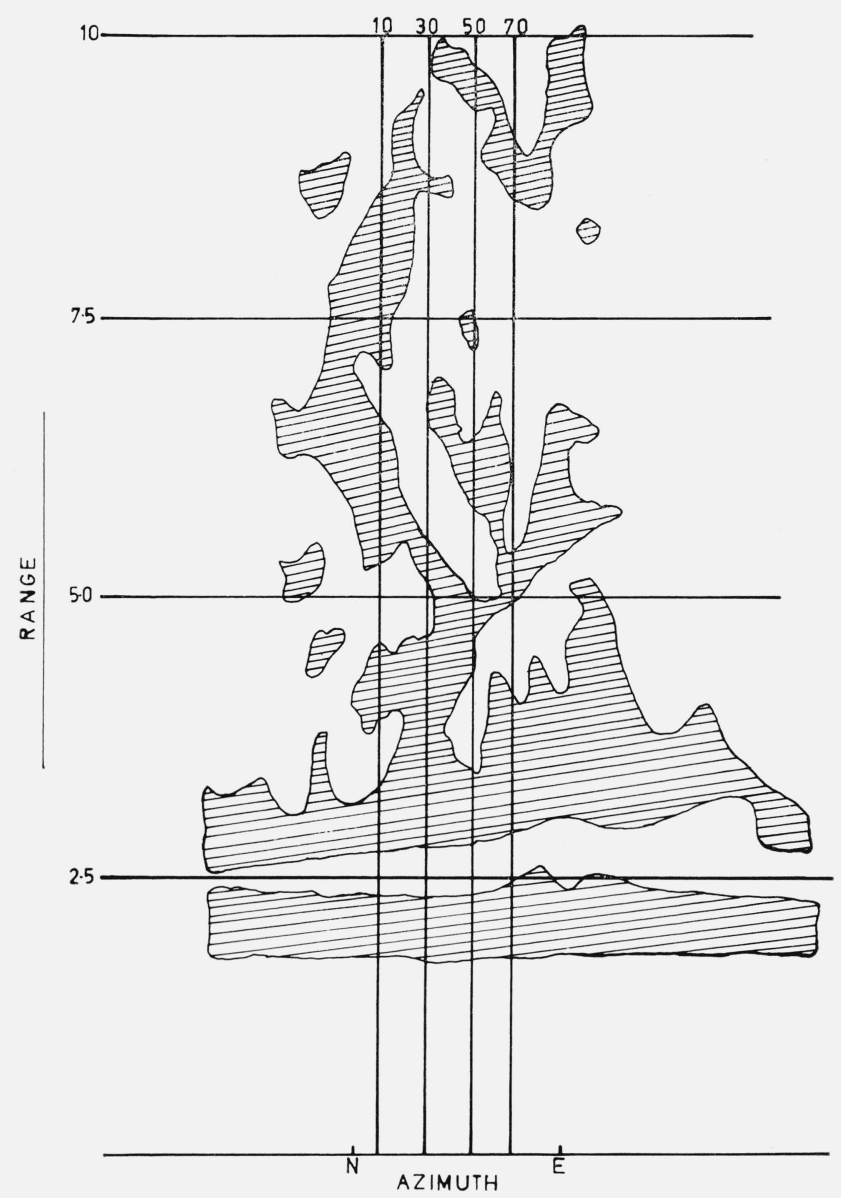

Figure 12. A tracing of the record for $1700 \mathrm{hr}$, 23d September, 1960 .

This reasonably typical record may be compared with the computed echo system of figure 11 .

It is rather difficult to determine an "average" backscatter range-azimuth diagram for any 1 month, as the daily variations are so great. However, a reasonably typical record is shown in figure 11 for comparison with the derived sections of figure 12 . The detailed comparison is poor, but the general features are in rough agreement.

\section{Conclusions}

The observations and the analyses presented in this paper, lead to several main conclusions.

(a) Using a rather simple method of projection of data, it has been shown that large ionospheric $F$-region tilts occur along great circle paths leaving Brisbane and passing through equatorial regions in early evening hours. These tilts (both positive and negative) range in angle from about $1^{\circ}$ to $6^{\circ}$. The time sequence of progression of the tilted region around the world agrees well with the time sequence of occurrence of anomalous transequatorial echoes with change in azimuth of observation.

(b) Profiles of the critical frequency of the $F$-region are an aid in accounting for secondary effects on the 
observed anomalous echo behavior, due to changes in propagation within the ionosphere rather than below it.

(c) Very pronounced modifications of the modes of propagation of HF signals, along paths traversing the equatorial regions in evening hours, are produced by quite small tilts in the ionospheric $F_{2}$ region.

(d) The more important of the transequatorial tilt-mode propagation paths occur at low elevation angles. A high degree of ray focusing commonly occurs for elevation angles below $8^{\circ}$, and in some cases for angles up to $18^{\circ}$. These focused modes lead to very stable backscatter echo configurations, such as are commonly observed for anomalous transequatorial echoes.

(e) In the analysis of backscatter propagation, due regard must be paid to the possibility of "mixedmode" propagation, in which energy traveling to the ground-scatter point by one mode may return to the receiver via any available mode. This effect is important for long-range echoes.

(f) The observed long-range backscatter echoes are thought to be composed of signals arriving via a number of propagation modes. One of these modes is often much more focused than the remainder and contributes greatly to the range stability of the various mixed-mode signals making up the echo.

(g) The relative signal strangths of observed longrange backscatter signals can only be reasonably explained if the effects of "horizon focusing" are taken into account. These same effects are important for all long-distance $\mathrm{HF}$ communications.

Thanks are due to the following Centers for supplying data used for the day of October 6, 1960: IGY World Data Center A, NBS Laboratories, Boulder, U.S.A. The Geophysical Observatory, Christchurch, New Zealand. The Ionospheric Prediction Service of Australia, Sydney, Australia. The Radio Research Laboratories, Tokyo, Japan. The University of São Paulo, São Paulo, Brazil. Additional median data was obtained from data tabulated in the CRPL F-series, Part A, publications.

Thanks are due to Professor H. C. Webster for his continued support. Mrs D. Neilson greatly assisted with the general data compilation and Mr. P. Dyson with the seasonal tilt analysis.

The work reported herein has been sponsored partly by the Electronics Research Directorate of the Air Force Cambridge Research Laboratories, Office of Aerospace Research under contract AF 64(500)-9, partly by the University of Queensland Research Committee, and partly by the Radio Research Board of the C.S.I.R.O.

\section{References}

Dueno, B. (1960), Peculiarities and seasonal variations of transequatorial backscatter echoes as observed at Mayagüez, Puerto Rico, J. Geophys. Res. 65, No. 6, 1691-1698.

Gerson, N. C. (1962), Radio wave absorption at long distances, J. Atmospheric Terrest. Phys. 23, No. 1, 113-125.

Kift, F. (1960), The propagation of high frequency radio waves to long distances, Proc. Inst. Elec. Engrs. 107B, No. 32, 127-140.

Martyn, D. F. (1955), Geomagnetic anomalies of the $F 2$ region and their interpretation, Physics of the Ionosphere, pp. 260-264 (The Physical Society of London, London S. W. 7).

Martyn, D. F. (1959), The normal $F$-region of the ionosphere, Proc. IRE 4\%, No. 2, 147-155.

Muldrew, D. B., and R. G. Maliphant (1962), Long distance one-hop ionospheric radio-wave propagation, J. Geophys. Res. 6\%, No. 5, 1805-1815.

Piggot, W. R. (1953), The reflection and absorption of radio waves in the ionosphere, Proc. Inst. Elec. Engrs. 100B, No. $1,61-72$.

Rawer, K. (1952), Radio wave absorption at long distances, Wireless Engr. 29, No. 350, 287-301.

Rawer, K., and E. Taieb (1960), Determination of electron density profiles in the ionosphere, Compt. Rend. 251, No. $4,576-578$.

Rossi, B. (1957), Optics, p. 57 (Addison Wesley, Cambridge, Mass.).

Shearman, E. D. R. (1956), The technique of ionospheric investigation using ground backscatter, Proc. Inst. Elec. Engrs. 103B, No. 8, 210-223.

Smith, E. K., Jr. (1957), Worldwide occurrence of sporadic $E$, N.B.S. Circ. No. 582 .

Stein, S. (1958), The role of ionospheric-layer tilts in long range high-frequency radio propagation, J. Geophys. Res. 63, No. 1, 217-241.

Thomas, J. A. (1961), Transequatorial backscatter observations of magnetically controlled ionization, Nature 191, No. $4790,792$.

Thomas, J. A., and B. A. McInnes (1962), Transequatorial propagation analysis: ray tracing and mode analysis, Scientific Report No. 10, Physics Department, University of Queensland, Brisbane, Australia.

Thomas, J. A., and R. W. E. MeNicol (1960), A highly directive rotating array for $16 \mathrm{Mc} / \mathrm{s}$, Nature 18\%, No. 4735, 398-399.

Villard, O. G., S. Stein, and K. C. Yeh (1957), Studies of transequatorial ionospheric propagation by the scattersounder method, J. Geophys. Res. 62, No. 3, 399-412.

Wright, J. W. (1960), A model of the $F$-region above $h_{\text {max }} F 2$, J. Geophys. Res. 65, No. 1, 185-191. 